OPEN ACCESS

Edited by:

Ilse Denise Jacobsen,

Leibniz-Institut für Naturstoff-

Forschung und Infektionsbiologie,

Hans Knöll Institut, Germany

Reviewed by: Agostinho Carvalho,

University of Minho, Portugal Sven Krappmann,

University of Erlangen-

Nuremberg, Germany

*Correspondence:

Juergen Loeffler

loefflerj@ukw.de

tThese authors have contributed equally to this work.

Specialty section:

This article was submitted to Microbial Immunology,

a section of the journal

Frontiers in Immunology

Received: 27 September 2017 Accepted: 21 November 2017

Published: 06 December 2017

Citation:

Hellmann A-M, Lother J, Wurster S, Lutz MB, Schmitt AL, Morton CO,

Eyrich M, Czakai K, Einsele $H$ and Loeffler J (2017) Human and Murine Innate Immune Cell Populations

Display Common and Distinct Response Patterns during Their

In Vitro Interaction with the Pathogenic Mold

Aspergillus fumigatus.

Front. Immunol. 8:1716. doi: 10.3389/fimmu.2017.01716

\section{Human and Murine Innate Immune Cell Populations Display Common and Distinct Response Patterns during Their In Vitro Interaction with the Pathogenic Mold Aspergillus fumigatus}

Anna-Maria Hellmann ${ }^{1 t}$, Jasmin Lother ${ }^{1 \dagger}$, Sebastian Wurster ${ }^{1}$, Manfred B. Lutz'2, Anna Lena Schmitt', Charles Oliver Morton ${ }^{3}$, Matthias Eyrich $^{4}$, Kristin Czakai', $^{1}$ Hermann Einsele ${ }^{1}$ and Juergen Loeffler ${ }^{1 *}$

${ }^{1}$ Medizinische Klinik \& Poliklinik II, Universitätsklinikum Würzburg, Würzburg, Germany, ${ }^{2}$ Institute of Virology and Immunobiology, University of Würzburg, Würzburg, Germany, ${ }^{3}$ School of Science and Health, Western Sydney University, Campbelltown, NSW, Australia, ${ }^{4}$ Kinderklinik und Poliklinik, Universitätsklinikum Würzburg, Würzburg, Germany

Aspergillus fumigatus is the main cause of invasive fungal infections occurring almost exclusively in immunocompromised patients. An improved understanding of the initial innate immune response is key to the development of better diagnostic tools and new treatment options. Mice are commonly used to study immune defense mechanisms during the infection of the mammalian host with A. fumigatus. However, little is known about functional differences between the human and murine immune response against this fungal pathogen. Thus, we performed a comparative functional analysis of human and murine dendritic cells (DCs), macrophages, and polymorphonuclear cells (PMNs) using standardized and reproducible working conditions, laboratory protocols, and readout assays. A. fumigatus did not provoke identical responses in murine and human immune cells but rather initiated relatively specific responses. While human DCs showed a significantly stronger upregulation of their maturation markers and major histocompatibility complex molecules and phagocytosed $A$. fumigatus more efficiently compared to their murine counterparts, murine PMNs and macrophages exhibited a significantly stronger release of reactive oxygen species after exposure to $A$. fumigatus. For all studied cell types, human and murine samples differed in their cytokine response to conidia or germ tubes of $A$. fumigatus. Furthermore, Dectin-1 showed inverse expression patterns on human and murine DCs after fungal stimulation. These specific differences should be carefully considered and highlight potential limitations in the transferability of murine host-pathogen interaction studies.

Keywords: murine model, humans, Aspergillus fumigatus, innate immune response, fungal infection

\section{INTRODUCTION}

Humans inhale hundreds of airborne fungal spores daily, including spores from the saprophytic mold Aspergillus fumigatus, which is ubiquitous in the environment. While the fungus rarely causes diseases in healthy individuals, disorders of the immune system are associated with a wide spectrum of Aspergillus-related diseases (1). Overshooting immune response to the fungus 
can lead to hypersensitivity syndromes such as allergic bronchopulmonary aspergillosis, whereas invasive aspergillosis (IA) is a major cause of morbidity and mortality in immunocompromised patients.

The mammalian immune system, evolving under continuous exposure to airborne fungal spores, possesses a vast arsenal of strategies to combat invading fungi including mediating tolerance to commensals and limiting hyperinflammation to prevent tissue damage. In the absence of an effective immune response, Aspergillus conidia swell and become invasive by germinating into the lung tissue and entering the blood stream. Alveolar macrophages act as the first line of defense in the airways by phagocytosis of conidia and secretion of pro-inflammatory cytokines and reactive oxygen species (ROS) (2). Neutrophil granulocytes [polymorphonuclear cells (PMNs)] also play a major role in the early immune defense against IA, as they are able to prevent germination and kill fungal hyphae through the release of ROS, phagocytosis, or formation of neutrophil extracellular traps. Dendritic cells (DCs) represent an important bridge between innate and adaptive immunity as they process fungal antigens and subsequently stimulate specific T-cells via antigen-presentation by major histocompatibility complex (MHC) I and II molecules. They also orchestrate the immune response by secreting an array of pro- and anti-inflammatory cytokines. Stimulation of pattern recognition receptors (PRRs), such as toll-like receptors (TLR)-2 and -4 (3) and the Dectin1 -receptor, is crucial to the activation of these immune cell subsets (4).

Detailed insights into the pathophysiology of the infection and mechanisms of the host-pathogen interaction are urgently needed to facilitate development of new prophylactic and therapeutic tools and strategies. Due to their easy accessibility, relatively short generation time, and availability of genetically defined strains, mouse models are commonly used to characterize the interaction of $A$. fumigatus with the mammalian host in vivo and to evaluate novel therapeutic strategies. There is, however, increasing evidence of major functional differences between the human and murine immune systems, indicating limited application of data obtained in studies employing murine cells or models $(5,6)$. On one hand, the composition of the murine leukocyte repertoire differs from that of humans; in mice only $7-28 \%$ of peripheral blood leukocytes are PMNs in contrast to $35-70 \%$ in human blood (7). On the other hand, functional differences, such as the sequence of participating immunological cells after antigen challenge as well as the dose of antigen needed to initiate immunological reaction in delayed type hypersensitivity have been described (5).

So far, little is known about functional differences in the human and murine immune defense against $A$. fumigatus. Thus, this study sought to provide a comparative functional assessment between human and murine innate immune cell subsets routinely employed in host-pathogen interaction studies (8-13). Our data demonstrate that mice and men which differ in their size, habitat, lifespan, genome size, and blood composition possess principle differences in how selected innate immune cell populations specifically interact with the pathogenic mold A. fumigatus.

\section{MATERIALS AND METHODS}

\section{Fungal Strains and Cultivation Conditions}

Aspergillus fumigatus strain American Type Culture Collection 46645 was incubated on beer wort agar (Institute of Hygiene and Microbiology, University of Wuerzburg, Germany) for $72 \mathrm{~h}$ at $37^{\circ} \mathrm{C}$. Conidial suspensions were prepared by rinsing plates with sterile water and filtered through a $40-\mu \mathrm{m}$ cell strainer (BD Falcon ${ }^{\mathrm{TM}}$ Cell Strainer, BD Biosciences). To generate germ tubes, $1 \times 10^{8}$ resting conidia were inoculated in Rosewell Park Memorial Institute (RPMI) 1640 Medium (Life technologies) and cultured at $37^{\circ} \mathrm{C}$ until the germ tubes reached a length of about 10-30 $\mu \mathrm{m}$. To preserve fungal morphotypes during coculture experiments, conidia and germ tubes were inactivated with $100 \%$ ethanol (Sigma) for $30 \mathrm{~min}$, washed five times with sterile water, and stored in RPMI at $-20^{\circ} \mathrm{C}$. For time-lapse microscopy red fluorescent $A$. fumigatus conidia and germ tubes were generated as described before (9).

\section{Isolation and Culture of Human and Murine Immune Cells Dendritic Cells}

Peripheral blood mononuclear cells were isolated from leukocyte concentrates from healthy human donors (Institute of Transfusion Medicine, University Hospital of Wuerzburg) by Ficoll (Biochrome) density centrifugation. After positive magnetic selection of CD14 ${ }^{+}$cells (CD14 MicroBeads, Miltenyi Biotec), monocytes were cultured for 5 days in RPMI 1640 medium supplemented with $10 \%$ fetal calf serum (FCS) (Sigma), rGM-CSF (100 ng/ml, Bayer), and rIL4 (10 ng/ml, Miltenyi Biotec) (9).

Murine bone marrow derived dendritic cells (BMDCs) were generated as described before (14). Briefly, bone marrow from healthy mice was harvested and cultured in R10-medium (RPMI 1640) (GIBCO BRL) with $100 \mathrm{U} / \mathrm{ml}$ Penicillin (Sigma), $100 \mu \mathrm{g} /$ $\mathrm{ml}$ Streptomycin (Sigma), $2 \mathrm{mM}$ L-glutamine (Sigma), $50 \mu \mathrm{M}$ 2-mercaptoethanol (Sigma), 10\% FCS (PAA), and $200 \mathrm{U} / \mathrm{ml}$ rmGM-CSF (Reprotech/Tebu) for 6 days. The non-adherent cells were harvested and cultured in R10-medium without cytokines.

\section{Polymorphonuclear Cells}

To isolate human PMNs anticoagulated blood from healthy human donors was layered on a polysaccharide gradient (Polymorphprep, Axis Shield). After $30 \mathrm{~min}$ of centrifugation $(500 \mathrm{~g})$, the PMN interphase was harvested and pelleted for $5 \mathrm{~min}$ at $300 \mathrm{~g}$. Remaining erythrocytes were lysed with EL buffer (Qiagen). Isolation of murine PMNs, which were obtained from bone marrow of $\mathrm{C} 57 \mathrm{BL} / 6 \mathrm{NCrl}$ mice, was performed using the EasySep ${ }^{\mathrm{TM}}$ Mouse Neutrophil Enrichment Kit (Stem Cell Technologies) according to the manufacturer's protocol. Both murine and human PMNs were cultured in RPMI 1640 Medium with $5 \%$ FCS.

\section{Macrophages}

Human monocytes obtained from healthy subjects as described above were cultured in RPMI 1640 medium supplemented with $10 \%$ FCS and rM-CSF (Immunotools) for 6 days. Only adherent 
cells were used for experiments. To generate murine macrophages, bone marrow cells of healthy mice were cultured in R10-medium with M-CSF supernatant [mouse M-CSF (Immunotools) supernatant, L929] for 6 days. Adherent cells were harvested and suspended in R10-medium.

\section{Time-Lapse Video Microscopy}

$3.5 \times 10^{4}$ human monocyte-derived immature dendritic cells (moDCs) or murine BMDCs were stimulated with $3.5 \times 10^{5}$ A. fumigatus (Afu-dTomato) conidia or germ tubes [multiplicity of infection $(\mathrm{MOI})=10$ ]. Polystyrene beads (Sigma) were used as an unspecific stimulus. Image acquisition over a time period of $3 \mathrm{~h}$ was performed using a Leica AF6000 time-lapse microscope with a picture frequency of $5 / \mathrm{min}$. Image analysis was conducted with LAS AF lite (Leica), ImageJ1.45s (Wayne Rasband), and Irfan View 4.32 (Irfan Skiljan) software. For each stimulus, phagocytosis activity was analyzed in six independent movies, respectively, following moDCs or BMDCs over a time period of $3 \mathrm{~h}$ by separately counting phagocytosed and extracellular particles or fungi, respectively.

\section{Flow Cytometry}

Dendritic cells were stimulated with inactivated A. fumigatus conidia and germ tubes (MOI = 1), $100 \mu \mathrm{g} / \mathrm{ml}$ zymosan depleted [a yeast cell wall preparation, which was treated with hot alkali to remove all TLR-stimulating properties to selectively activate Dectin-1 (dZym, InvivoGen)] or $1 \mathrm{mg} / \mathrm{ml}$ lipopolysaccharid (LPS, Sigma) for $24 \mathrm{~h}$. Subsequently, cells were harvested, washed, and resuspended in cold Hank's balanced salt solution (HBSS, Sigma) containing 2 mM EDTA (Sigma). The following antibodies were used for extracellular staining: HLA-ABC-PE (BD Biosciences), HLA-DR-PE (BD Biosciences), CD1a-APC (BD Biosciences), CD14-FITC (BD Biosciences), CD80-APC (Miltenyi Biotec), CD86-PE (BD Biosciences), Dectin-1-PE (R\&D) (human cells) and HLA-2K $\mathrm{K}^{\mathrm{b}}$-FITC (BD Biosciences), HLA-Ia/I-E-PE (BioLegend), CD11c-APC (BioLegend), CD80FITC (BioLegend), CD86-PE (BD Biosciences), and Dectin-1-PE (R\&D) [murine cells]. $5 \times 10^{5}$ cells were stained for $15 \mathrm{~min}$ at $4^{\circ} \mathrm{C}$. Subsequently, cells were washed twice and $10^{4}$ viable cells according to FSC-SSC properties were acquired using a FACS Calibur (BD Biosciences) flow cytometer and CellQuest Pro software (version 5.2). Data were analyzed with FlowJo software (Tree Star Inc., Ashland, OR, USA).

\section{ROS Quantification}

Polymorphonuclear cells and macrophages were stimulated with inactivated $A$. fumigatus conidia and germ tubes $(\mathrm{MOI}=1)$, $100 \mu \mathrm{g} / \mathrm{ml}$ zymosan depleted or $10 \mu \mathrm{g} / \mathrm{ml}$ phorbol-myristateacetate [the major endogenous ROS inducer in PMNs and macrophages (PMA, Sigma-Aldrich)]. ROS-formation in human and murine PMNs and macrophages was determined by ROSdependent $\mathrm{CM}-\mathrm{H}_{2} \mathrm{DCF}$ oxidation (dichlorfluorescein, SigmaAldrich). Excitation was performed at $485 \mathrm{~nm}$, and fluorescence emission was detected at $535 \mathrm{~nm}$ (GENios microplate reader, TECAN). PMNs were stimulated at $37^{\circ} \mathrm{C}$ for $1 \mathrm{~h}$ and macrophages for $2.5 \mathrm{~h}$ before measuring fluorescence intensity.

\section{Multiplex Cytokine Assays}

Polymorphonuclear cells $\left(2 \times 10^{6} / \mathrm{ml}\right)$, macrophages $\left(8 \times 10^{5} / \mathrm{ml}\right)$, and DCs $\left(1 \times 10^{6} / \mathrm{ml}\right)$ were stimulated with $A$. fumigatus conidia and germ tubes (MOI $=1)$, zymosan depleted $(100 \mu \mathrm{g} / \mathrm{ml})$, LPS $(1 \mu \mathrm{g} / \mathrm{ml})$, or plain culture medium (negative control). Supernatants were harvested after $3 \mathrm{~h}$ (PMNs), $12 \mathrm{~h}$ (macrophages), or $24 \mathrm{~h}$ (DCs) and stored at $-20^{\circ} \mathrm{C}$. Cytokine concentrations (IL1 $\beta$, IL4, IL6, huIL8, muGroo, IL10, IL12p70, IL18, IL23, IP10, MCP1, MIP1 $\alpha$, MIP1 $\beta$, TNF $\alpha$ ) were quantified with a human and mouse 13-Plex panel assay (Affymetrix, eBioscience).

\section{Fungicidal Activity of PMNs}

Polymorphonuclear cell were isolated from whole blood of four healthy human donors and two mice as described above. PMNs were diluted in colorless RPMI $+10 \%$ FCS at a concentration of $2 \times 10^{6} / \mathrm{ml} .5 \times 10^{5} \mathrm{PMNs}$ were seeded per well of a 24 -well plate and $5 \times 10^{5}$ vital $A$. fumigatus germ tubes were added $(\mathrm{MOI}=1$, ideal MOI was determined in a preceding experiment). Control wells containing only PNMs or fungal cells as well as blank wells containing culture medium without cells were prepared. After 2 and $4 \mathrm{~h}$ of coculture at $37^{\circ} \mathrm{C}$, hypotonic lysis of PMNs was performed by washing wells twice with $1,000 \mu \mathrm{l}$ cold distilled water, followed by 5 min incubation on ice. Supernatants were carefully removed, and $200 \mu \mathrm{l}$ HBSS supplemented with $400 \mu \mathrm{g} / \mathrm{ml}$ of 2,3-bis-(2-methoxy-4-nitro-5-sulphenyl)-(2H)tetrazolium-5-carboxanilide [XTT (Sigma)] and $50 \mu \mathrm{g} / \mathrm{ml}$ of coenzyme (Sigma) were added. After $90 \mathrm{~min}$ incubation at $37^{\circ} \mathrm{C}$ and centrifugation at $300 \mathrm{~g}$ for $5 \mathrm{~min}, 100 \mu \mathrm{l}$ supernatant of each well were transferred to a 96 -well plate and $\mathrm{OD}_{450}$ was measured in a microplate reader. Fold changes of fungal XTT metabolism were calculated according to the following formula: Fold change $=\left(\mathrm{OD}_{450}^{\text {PMN+Fungus }}-\mathrm{OD}_{450}^{\mathrm{PMN}}\right) /\left(\mathrm{OD}_{450}^{\text {Fungus }}-\mathrm{OD}_{450}^{\text {Blank }}\right)$.

\section{Statistics}

Significance testing was performed using GraphPad Prism 7 (Graphpad Software, Inc.) using different statistical test which are noted in each figure legend. Statistical significance is denoted as follows: ${ }^{*} p<0.05,{ }^{* *} p<0.01$, and ${ }^{* *} p<0.001$.

\section{RESULTS}

\section{Murine Neutrophils and Macrophages Show Stronger ROS Release upon Stimulation with $A$. fumigatus Germ Tubes and Depleted Zymosan (dZym)}

Reactive oxygen species production by human and murine neutrophils and macrophages cocultured with A. fumigatus conidia or germ tubes was quantified and compared. No significant induction of oxidative burst in PMNs or macrophages of either species was observed upon conidial stimulation. A. fumigatus germ tubes and dZym (binding exclusively to dectin-1) led to significantly higher ROS release by murine PMNs (Figure 1A) and macrophages (Figure 1B). By contrast, human PMNs and 

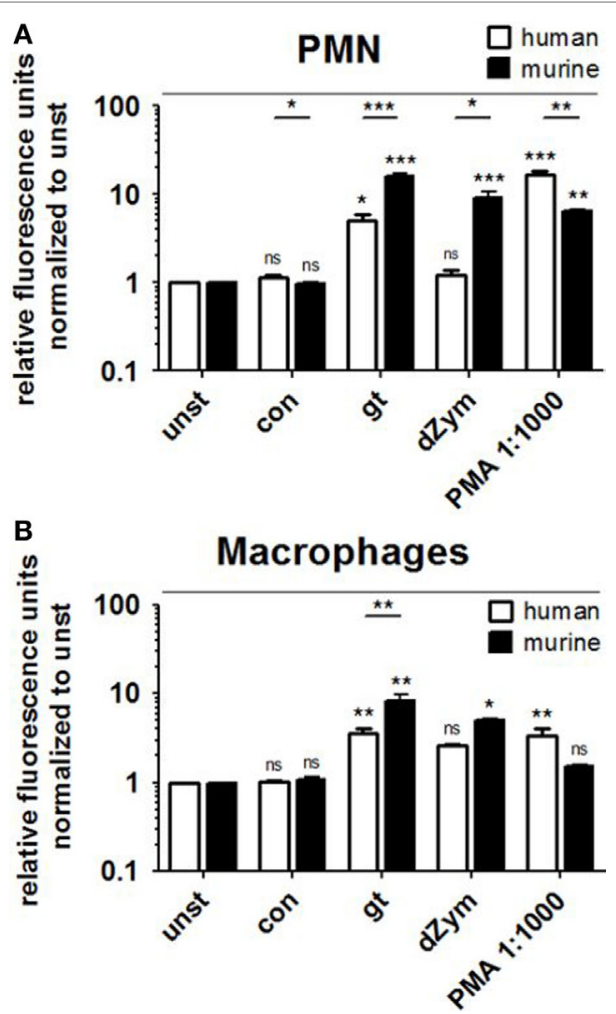

FIGURE 1 | Murine polymorphonuclear cells (PMNs) and macrophages exhibit a stronger reactive oxygen species (ROS) response to Aspergillus fumigatus germ tubes and depleted zymosan (dZym). Human and murine PMNs (A) or macrophages (B) were stimulated with $A$. fumigatus conidia (con), germ tubes (gt), dZym, or phorbol 12-myristate 13-acetate (PMA). ROS production was measured by a dichlorfluorescein-based assay. Mean fold changes of relative fluorescence units after 2 (PMNs) and $3 \mathrm{~h}$ (macrophages) are shown. Seven human and three murine samples were analyzed. Significance was calculated as difference to unstimulated with ANOVA and Tukey post-test (stars above of bars) as well as between human and mouse specimens with unpaired $t$-Test, Error bars indicate SDs.

macrophages released significantly more ROS after stimulation with the major endogenous ROS inducer PMA.

\section{Murine PMNs Exert Stronger and Broader Pro-inflammatory Cytokine Response to A. fumigatus Germ Tubes and dZym}

Strong ROS response was paralleled by the secretion of proinflammatory cytokines Gro $\alpha$, IL6, MIP1 $\alpha$, MIP $1 \beta$, and TNF $\alpha$ by murine PMNs after stimulation with $A$. fumigatus germ tubes or dZym, as well as the anti-inflammatory cytokine IL10 (Figure 2). Pro-inflammatory cytokine release was also significantly induced by PMA stimulation, whereas coculture with $A$. fumigatus conidia did not result in significant induction of MIP $1 \beta$ or TNF $\alpha$ secretion. Human PMNs showed strongly elevated IL8 release when stimulated with $A$. fumigatus germ tubes, dZym or PMA, while MIP $1 \alpha$ and MIP $1 \beta$ secretion was weakly induced by A. fumigatus germ tubes and dZym. $\square$ human

murine

A IL8/Gro $\alpha$
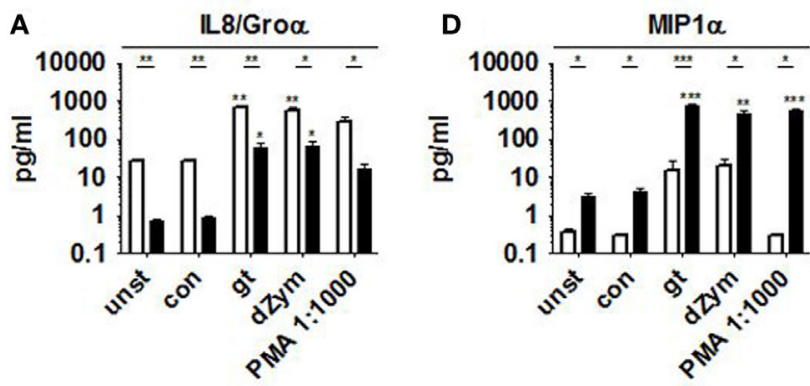

B

IL6

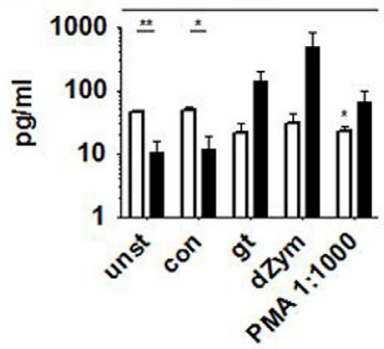

E
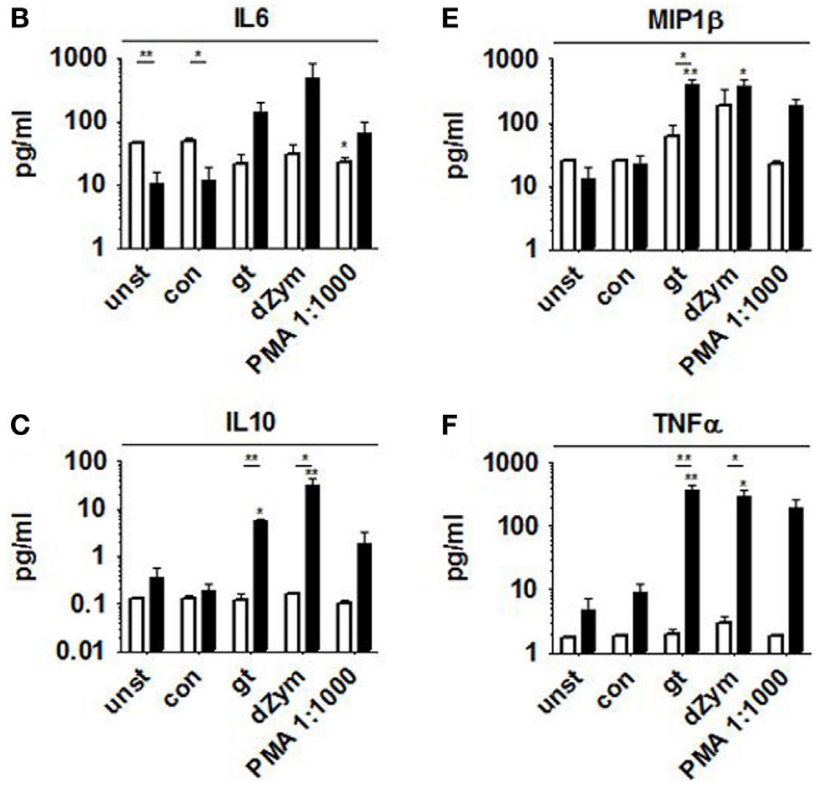

FIGURE 2 | Aspergillus fumigatus germ tubes and depleted zymosan (dZym) trigger strong pro-inflammatory cytokine release by murine neutrophils.

Murine or human neutrophils were stimulated with $A$. fumigatus conidia, germ tubes, dZym or PMA for a period of $3 \mathrm{~h}$. Subsequently, cytokine concentrations in the culture medium were determined by 13-plex cytokine assays. PMNs obtained from three human donors (white bars) and three mice (black bars) were assessed. Mean concentrations of IL8/Gro $\alpha$ (A), IL6 (B), IL10 (C), MIP1 $\alpha$ (D), MIP1 $\beta$ (E), and TNF $\alpha$ (F) are shown. Error bars indicate standard deviations. Significance was calculated as difference to unstimulated with ANOVA and Tukey post-test (stars above of bars) as well as between human and mouse specimens with unpaired $t$-Test.

\section{Human and Murine Macrophages Release Distinct Cytokine Patterns When Cocultured with $A$. fumigatus or Stimulated with Synthetic Agonists}

Pro- and anti-inflammatory cytokine secretion was strongly induced in both murine and human macrophages upon coculture with $A$. fumigatus germ tubes, dZym, or synthetic agonists LPS and Pam3CSK. While human macrophages released higher concentrations of MIP1 $\alpha$, IL8, and IL10 (Figures 3B,D,E), fold changes of IL8 and MIP1 $\alpha$ were higher in murine samples due to differences in baseline levels. In comparison to murine cells stimulation of human macrophages by A. fumigatus germ tubes, 
$\mathrm{dZym}$, and Pam3CSK resulted in significantly greater IL1 $\beta$ release (Figure 3A). Interestingly, a small but significant increase in MIP1 $\alpha$ and TNFa (Figures 3E,F) secretion by macrophages of both species was observed upon conidial stimulation. In human macrophages, this was paralleled by an even more pronounced upregulation of IL8 release and minor elevations of IL1 $\beta$ and IL6 (Figures 3A,C,D).

\section{Human moDCs Show Higher Phagocytosis Rates of $A$. fumigatus Conidia and Germ Tubes}

Live imaging was performed to visualize the interaction between human (Figure 4A) or murine DCs (Figure 4B) with A. fumigatus conidia or germ tubes. Analyzing the number of touches of DCs and fungal cells or the unspecific polystyrene bead control revealed no significant differences between human and murine

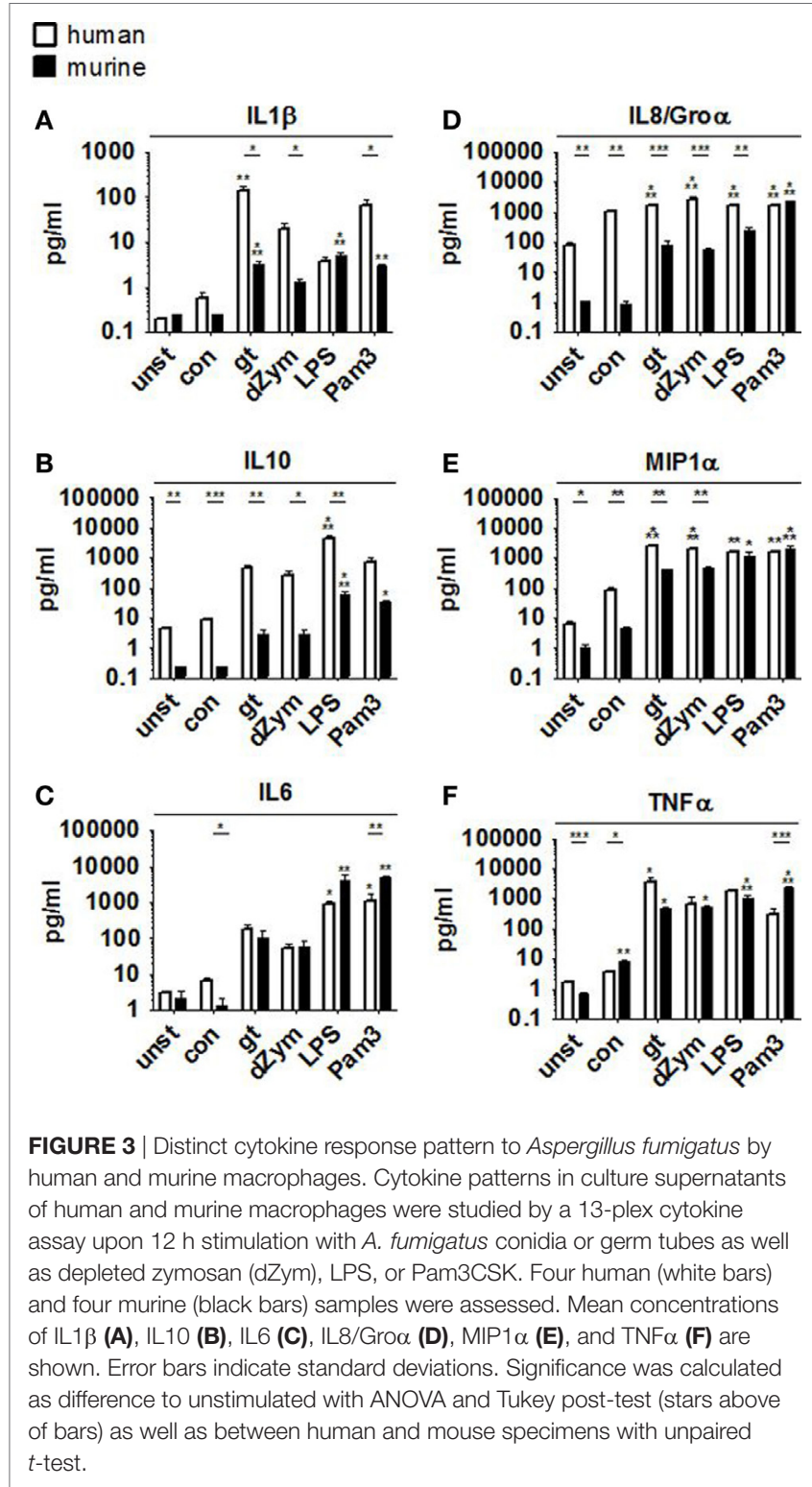

cells over $3 \mathrm{~h}$ (Figure 4C). Assessing the percentage of phagocytosed A. fumigatus conidia or germ tubes after $3 \mathrm{~h}$, however, human moDCs showed higher phagocytosis rates than murine BMDCs, whereas the internalization of polystyrene beads by human and murine DCs did not differ significantly (Figure 4D).

\section{Dectin-1 Is Inversely Regulated on Human and Murine DCs after Stimulation with \\ A. fumigatus Conidia and Germ Tubes}

The transmembrane glycoprotein Dectin-1, also known as C-type lectin domain family member 7A, plays an important role as an A. fumigatus phagocytosis receptor (4). Thus, Dectin-1 expression on DCs cocultured with ethanolinactivated conidia and germ tubes for different periods was assessed and compared with unstimulated DCs. After only $1 \mathrm{~h}$ of coculture with both fungal morphotypes, surface expression of Dectin-1 started to decline on human moDCs, with a more pronounced effect after stimulation with germ tubes (Figure 5A). By contrast, increased Dectin-1 expression was observed on murine BMDCs stimulated with A. fumigatus germ tubes (Figure 5B). For each of the coculture periods studied, a significant difference in Dectin-1 surface expression was detected between human and murine DCs stimulated with A. fumigatus germ tubes, whereas prolonged exposure $(18 \mathrm{~h})$ to inactivated conidia was necessary to observe significantly different expression (Figures 5C,D).

\section{Human and Murine DCs Cocultured with A. fumigatus Show Distinct Surface Antigen and Cytokine Response Patterns}

To further characterize the response patterns of human and murine DCs confronted with resting and germinated stages of A. fumigatus, we analyzed the expression of maturation markers (CD80 and CD86) and MHC molecules (MHC-I and MHC-II) on the cell surface. $24 \mathrm{~h}$ of stimulation with inactivated A. fumigatus germ tubes, dZym, and LPS led to 1.3-fold upregulation of CD80, CD86, MHC-I, and MHC-II on both human and murine DCs (Figure 6). Upon coculture with all studied stimuli, significantly greater levels of CD80 and CD86 were observed on human moDCs (Figures 6A,B). Coculture with A. fumigatus germ tubes led to a significantly higher MHC-I and MHC-II surface expression on human moDCs, whereas conidial stimulation resulted in stronger upregulation of MHC-I in murine BMDCs (Figures 6C,D).

Further, cytokine profiles were studied by bead-based multiplex cytokine assays after $24 \mathrm{~h}$ coculture of DCs with A. fumigatus conidia, germ tubes, dZym, or LPS. Stimulation with A. fumigatus germ tubes or LPS led to release of a broad range of pro-inflammatory cytokines by both human moDCs and murine BMDCs. Human moDCs showed significantly stronger $\mathrm{TNF} \alpha$ release in response to these stimuli and a trend toward stronger IL12p70 and IL23 induction. By contrast, murine BMDCs released significantly greater amounts of IL6 and IL18 (Figure 7).

Conidial stimulation resulted in the release of fewer cytokines and lower concentrations than other stimuli. Murine BMDCs 

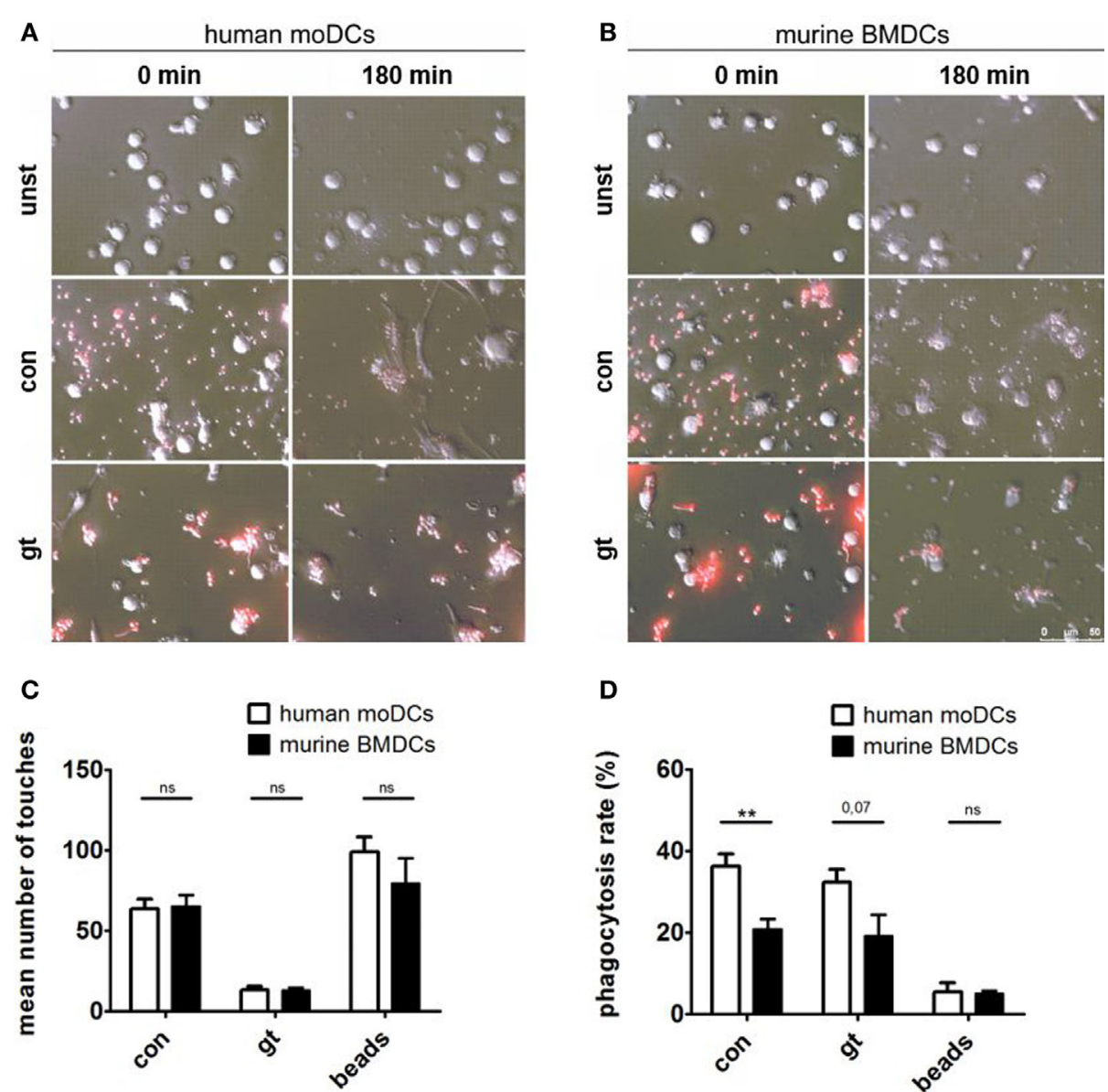

FIGURE 4 | Human dendritic cells (DCs) contact and phagocytose Aspergillus fumigatus conidia and germ tubes more effectively. Representative images show phagocytosis of red fluorescent conidia and germ tubes by human (A) and murine (B) DCs at the indicated time points (duration of coculture). Number of touches (C) of human and murine DCs with A. fumigatus conidia (con), germ tubes (gt), and polystyrene beads (beads) and phagocytosis rates (D) were determined by live imaging analysis. Mean values of touched (C) or mean percentage of phagocytosed (D) fungal cells or beads after $3 \mathrm{~h}$ of coculture are given in the figure. Six human and murine donors were assessed. Significance was calculated with unpaired $t$-Test and error bars indicate SDs.

showed strong and significant induction of the pro-inflammatory cytokines IL1 $\beta$, IL6, TNF $\alpha$, but also increased IL10 release after coculture with conidia. Stimulation of murine BMDCs with dZym led to significantly greater release of IL6, IL10, and IL18, whereas a tendency toward greater $\mathrm{TNF} \alpha$ release by human moDCs was observed.

Taken together, these results highlight different response patterns of human moDCs and murine BMDCs confronted with A. fumigatus, with distinct cytokine profiles and stronger expression of maturation markers and MHC molecules on human moDCs.

\section{Fungicidal Activity of PMNs}

Colorimetric analysis of the fungal metabolism revealed that $2 \mathrm{~h}$ of $A$. fumigatus germ tube-PMN co-cultivation led to markedly increased fungal metabolism while fungal metabolic activity was decreased again after $4 \mathrm{~h}$, compared to unstimulated control samples. No major difference was observed between human and murine PMNs (Figure S2 in Supplementary Material).

\section{DISCUSSION}

Aspergillus fumigatus is the most important cause of invasive fungal infections occurring almost exclusively in immunocompromised patients. A key to understanding A. fumigatus pathogenicity is knowledge of the interplay between the fungus and the immune system, in particular with the initial innate immune defense consisting of neutrophils, macrophages, and DCs.

In recent decades, several hundred studies characterized the functions of innate immune cells directed against $A$. fumigatus thereby improving the management and treatment of aspergillosis (15). This intensive work was based on in vitro studies involving culturing of primary human immune cells, and cell lines and a large variety of animal models including mice, which are frequently used to model human disease and to mimic scenarios of immunocompromised patients.

This study, the first to our knowledge, provides a comparative functional assessment of murine innate immune cell subsets routinely employed in A. fumigatus in vitro interaction studies with 


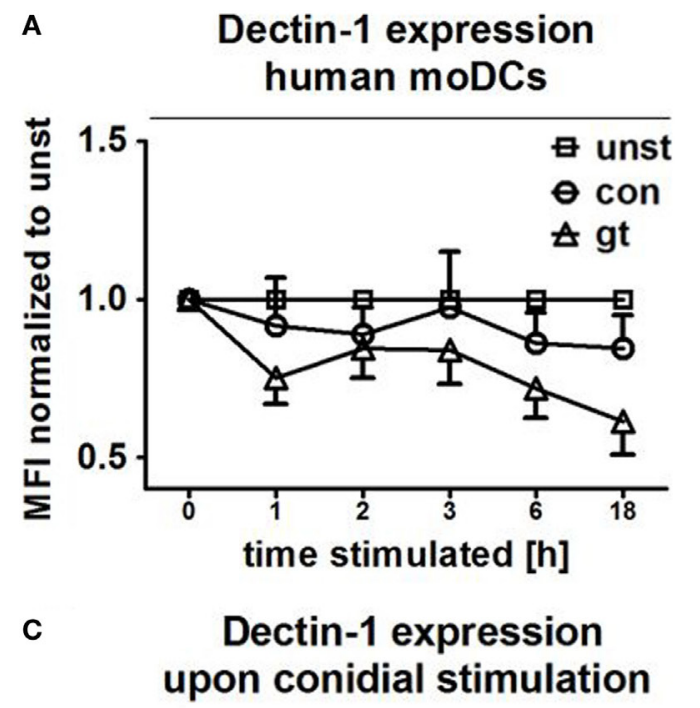

\section{B \\ Dectin-1 expression murine BMDCs}

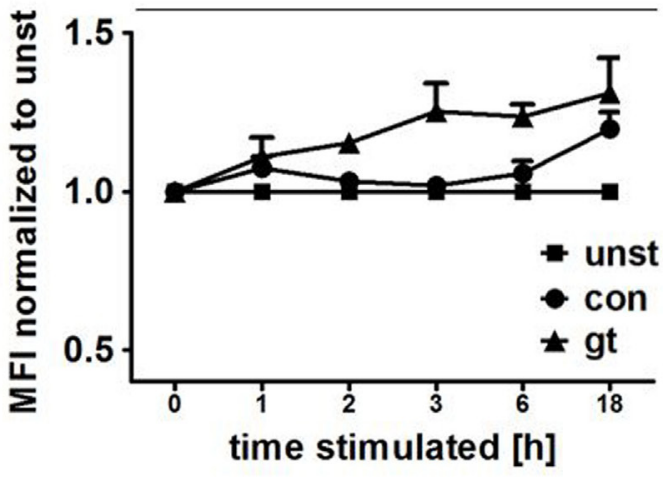

D

Dectin-1 expression upon germ tube stimulation

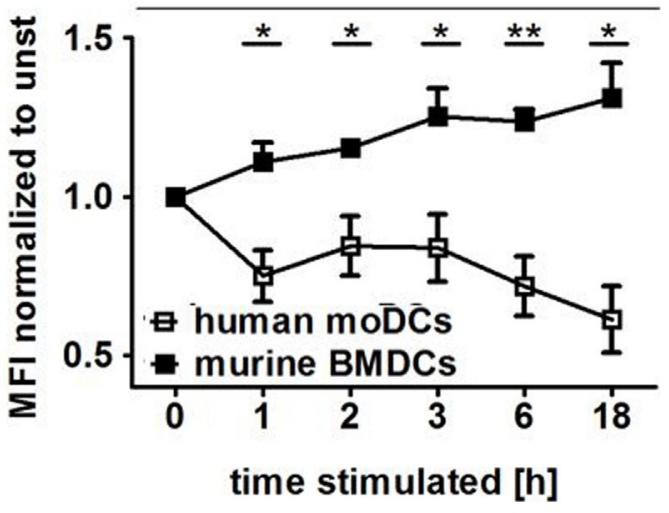

FIGURE 5 | Human and murine dendritic cells show inverse Dectin-1 surface expression patterns after exposure to Aspergillus fumigatus. Dectin-1 expression on the cell surface of human moDCs (A) and murine bone marrow derived dendritic cells (BMDCs) (B) was analyzed by flow cytometry after coculture with $A$. fumigatus conidia and germ tubes for different periods. Mean fold changes ( \pm SDs) of mean fluorescence intensity (MFI) compared to unstimulated cells are given in the figure. Two murine and four human samples were analyzed. The lower panels directly compare Dectin-1 expression following exposure of human moDCs and murine BMDCs to A. fumigatus germ tubes (C) and conidia (D). Human and murine samples were compared with unpaired $t$-Test, error bars show SDs.

their human counterparts. Humans and mice differ greatly in their size, lifespan, living conditions, and ecological niches. Even the blood composition between different mouse strains varies widely, while C57BL/6 mice contain 10-25\% PMNs and 75-90\% lymphocytes (16), CD-1 mice exhibit 15-20\% PMNs (300-2,000 cells/ $\mu \mathrm{l}$ ) and $50-70 \%$ lymphocytes $(1,000-7,000$ cells/ $\mu \mathrm{l})(17)$. In contrast, human blood contains 50-70\% PMNs $(3,500-7,000$ cells/ $\mu \mathrm{l})$ and $20-40 \%$ lymphocytes $(1,400-4,000$ cells/ $\mu \mathrm{l})(17)$. In consequence, a direct translation of murine in vivo experimental data to human pathological events often fails due to insufficient similarities in the organization of the immune system of both species (18).

In our study, we isolated and cultivated PMNs, macrophages, and DCs from both mammals and challenged them in vitro with A. fumigatus using standardized and reproducible working conditions, laboratory protocols and readout assays. We are aware that this in vitro comparison is restricted due to the isolated use of single immune cell types while well-established murine models allow a complex view of the pathogenesis of IA. However, the chosen comparative in vitro study design nicely illustrates the substantial parallel organization of the human and murine immune response against $A$. fumigatus but also provides examples for functional heterogeneity in the defense against the fungus.

Polymorphonuclear cells are among the first line response against $A$. fumigatus. When attracted by chemokines they leave the blood stream and migrate to the site of infection using a large number of PRRs to recognize and respond to the fungus. This includes the release of soluble antimicrobials, reactive metabolites, cytokines, and phagocytosis of conidia (19). In our experiments, murine and human neutrophils were directly isolated from anticoagulated blood using similar protocols (polysaccharide gradient for human blood and the commercial neutrophil enrichment kit for mice). Murine PMNs exhibited a significantly stronger ROS response to A. fumigatus and dZym, 


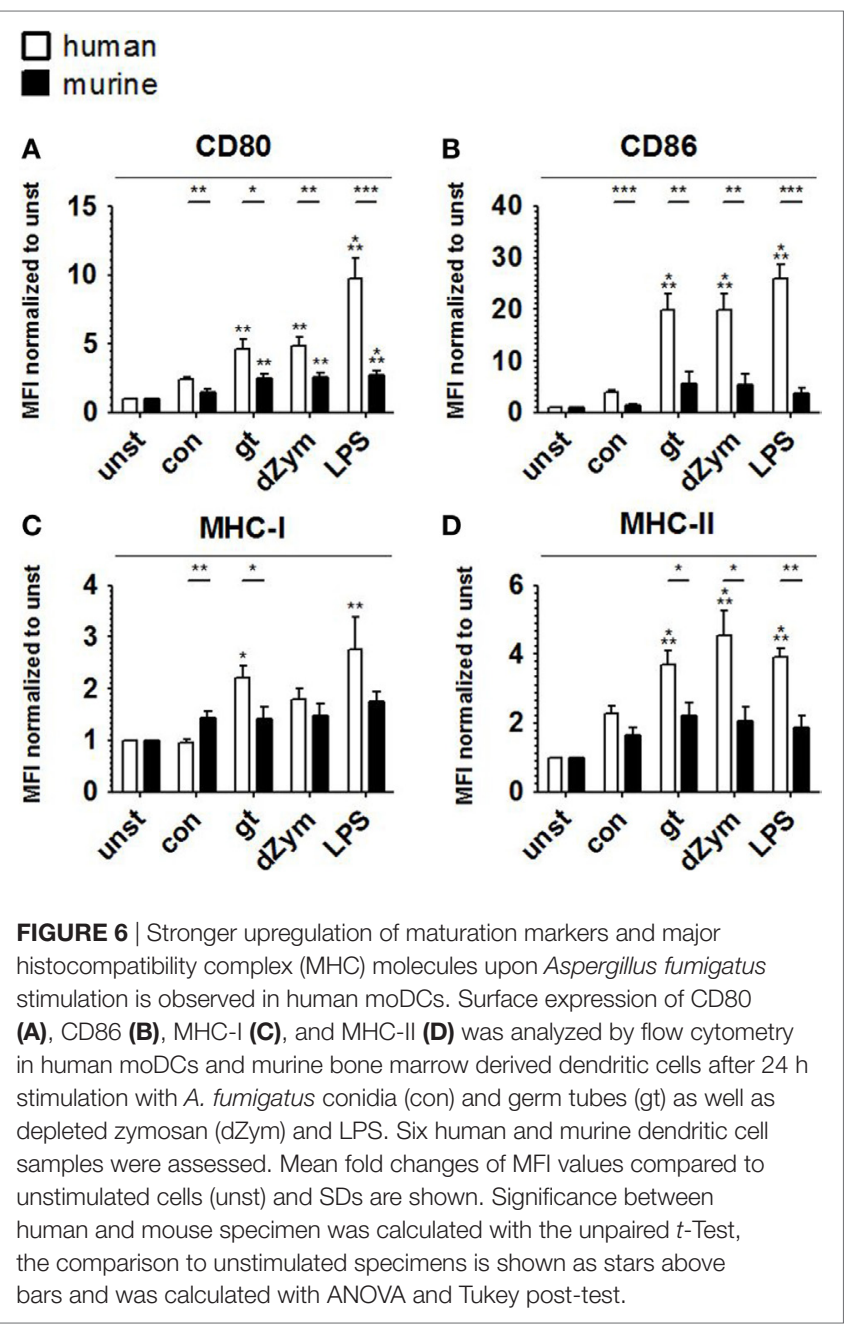

a pure dectin-1 agonist, than human PMNs (Figure 1). The role of ROS in the immune defense against A. fumigatus is rather unclear. While text-book knowledge indicates that ROS is used by PMNs to kill A. fumigatus, more recent reports state that they play a regulatory role as signaling molecules and in the activation of antimicrobial enzymes in the phagolysosome (20). Furthermore, it could be demonstrated only recently that murine lung PMNs trigger programmed cell death with apoptosis-like features in A. fumigatus conidia (21).

Furthermore, human PMNs showed strongly elevated IL8 release when stimulated with $A$. fumigatus germ tubes and dZym while murine PMNs secreted a much larger variety of cytokines and chemokines, including Groo, IL6, MIP1 $\alpha$, MIP1 $\beta$, and TNF $\alpha$, as well as the anti-inflammatory cytokine IL10. While IL8 is the most important chemoattractant for human neutrophil recruitment, an orthologous counterpart is absent in mice (22). In contrast, mice express CXCL15 as an attractant and other functional homologs of IL8, such as Groo (23).

Interestingly, in a preliminary experiment analyzing PMNs from four healthy human donors and two mice by an XTT metabolic assay, we revealed no major differences quantifying fungal metabolism after 2 and $4 \mathrm{~h}$, respectively. We hypothesize that the increase of fungal metabolism after $2 \mathrm{~h}$ of co-cultivation with human and murine PMNs might reflect temporarily higher fungal metabolic activity due to early defense mechanisms of A. fumigatus.

Human moDCs were generated from $\mathrm{CD} 14^{+}$monocytes using rGM-CSF and rIL4 while murine DCs were derived from bone marrow cultured in R10-medium supplemented with rmGM-CSF. While murine Ly6 $\mathrm{C}^{\text {high }}$ monocytes fail to proliferate (24), proliferating cells mostly represent macrophage-DC progenitors and common monocyte progenitor (25). This observation was described for human $\mathrm{CD} 14^{+}$monocytes undergoing moDC differentiation as well (26). Thereby, GM-CSF has major effects on myelomonocytic cells leading to a massive expansion of macrophage-DC-restricted precursors, but very low effects on common dendritic cell precursors (24). In both, humans and mice, Ly6 $\mathrm{C}^{\text {high }}$ monocytes and moDCs are recruited into tissue under inflammatory and infectious conditions thereby initiating $\mathrm{T}$ cell priming in the draining lymph nodes, e.g., in the synovia of rheumatoid arthritis patients $(27,28)$. Thus, murine BMDCs and human moDCs are highly similar and thus can be considered as functional homologs $(29,30)$.

Both, human and murine DCs were able to phagocytose A. fumigatus conidia and germ tubes. Efficient phagocytosis is a prerequisite for adequate antigen processing and presentation to lymphocytes (31). This characteristic property of DCs in the interplay with $A$. fumigatus underlines their relevance in bridging innate and adaptive immunity during mold infections. The more efficient phagocytosis of A. fumigatus conidia in human moDCs might be due to the differential size of human and murine DCs or due to mouse-strain specific variabilities in phagocytosic capacity (32).

Human moDCs secreted substantially more of the proinflammatory mediators TNF $\alpha, \mathrm{MCP} 1, \mathrm{IL} 12 \mathrm{p} 70$, and IL1 $\beta$, indicating their profound role in the broad activation of the innate and adaptive immune response. Murine BMDCs released significantly greater amounts of IL6 and IL18. Cenci et al. showed that IL6(-/-) mice were more susceptible to aspergillosis than the wild-type. Susceptibility was associated with increased inflammatory pathology, decreased antifungal effector functions of phagocytes, and impaired development of protective type 1 responses. Exposure to exogenous IL6 restored antifungal effector activity (33). Similarly, simultaneous neutralization of IL18 and IL12 resulted in a significant increase in A. fumigatus CFU in murine lung tissue demonstrating its key role in murine defense against Aspergillus infection (34).

Secretion of IL10, which suppresses proliferation, cytokine secretion, and costimulatory molecule expression of proinflammatory immune cells, differed markedly between human and murine cells. While A. fumigatus germ tubes and dZym led to a 100 - and 60 -fold induction of the IL10 secretion of human macrophages compared to unstimulated control cells, stimulation of murine macrophages led to lower increases (with germ tubes 14-fold, with dZym 14-fold). Interestingly, basal secretion of IL10 from unstimulated macrophages into the culture medium was over 20-fold higher in human cells than in their murine counterparts. Human and mouse IL10 have roughly $73 \%$ sequence homology and are secreted as 178 -amino acid proteins (35). It is 


\section{$\square$ human murine}
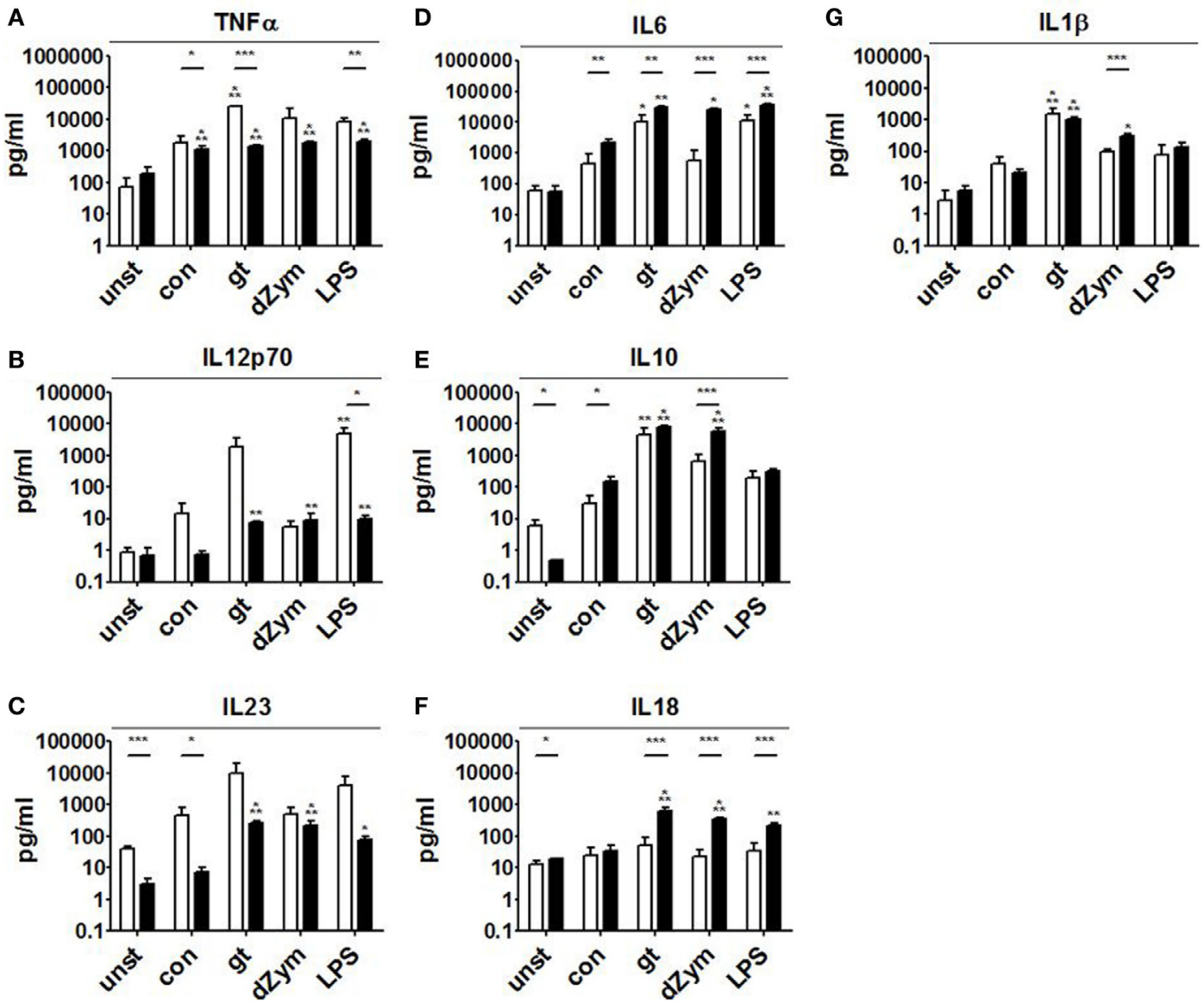

FIGURE 7 | Distinct cytokine response patterns to Aspergillus fumigatus are observed in human and murine dendritic cells (DCs). Cytokine concentrations (picograms/milliliter) in culture supernatants of human moDCs and murine bone marrow-derived dendritic cells stimulated with ethanol-inactivated $A$. fumigatus conidia or germ tubes, depleted zymosan (dZym) or LPS were quantified by 13-plex cytokine assays. Four human (white bars) and four murine (black bars) DC samples were assessed. Mean concentrations of TNF $\alpha$ (A), IL12p70 (B), IL23 (C), IL6 (D), IL10 (E), IL18 (F), and IL1 $\beta$ (G) are shown. Error bars indicate standard deviations. Significance was calculated as difference to unstimulated specimens with ANOVA and Tukey post-test (stars above of bars) as well as between human and mouse specimens with unpaired $t$-test.

unknown whether both cytokines have identical binding capacity to their receptors, stability, and degradation behavior.

Dectin-1, a $\beta$-glucan receptor, is expressed on monocytes, macrophages, DCs, neutrophils, and eosinophils (36). Brown et al. demonstrated that dZym, which was treated with hot alkali to remove its TLR-stimulating properties, triggers expression of pro-inflammatory cytokines via Dectin-1 signaling (37). In mice, sensing of $C$. albicans by Dectin- 1 results in ingestion and killing of the fungus and the induction of an early inflammatory response, which results in the recruitment and activation of other immune cells to the infection site. Dectin-1 deficiency leads to increased susceptibility to systemic and mucosal candidiasis (38). In contrast, human Dectin-1 deficiency causes diminished cytokine responses, while alternative receptors are then responsible for fungal uptake and subsequent killing. Our results demonstrate inverse Dectin-1 surface expression after exposure to
A. fumigatus. After $1 \mathrm{~h}$ of coculture, surface expression of Dectin-1 decreased on human moDCs, with a more pronounced effect after stimulation with germ tubes (Figure 5A). By contrast, increased Dectin-1 expression was observed on murine BMDCs stimulated with A. fumigatus germ tubes (Figure 5B). We hypothesize that downregulation of Dectin-1 exposure on human moDCs prevents severe and uncontrolled inflammatory reactions while on murine BMDCs, Dectin-1 expression is required for continuous ingestion and killing of the fungus. This is consistent with our previous data showing that anti-Dectin-1 antibody treatment of human moDCs as well as ex vivo myeloid DCs subsequently inhibited secretion of pro-inflammatory cytokines after contact with A. fumigatus, C. albicans, or zymosan $(4,39)$. This is also consistent with the observation that naïve mice lacking Dectin-1 show reduced killing of conidia and a higher mortality rate than wild-type mice (40). 
Taken together, after 65 million years of independent evolution (5), immune systems of both species differ substantially. Mice are perfectly adapted to their relatively short lifespan of 2-3 years and their natural habitat on the ground. In the defense against invading fungi, resistance mechanisms and an efficient control over pathogens dominate in humans, while tolerance determines the immune response in mice (41). Our comparative functional assessment of selected human and murine innate immune cell subsets using standardized and reproducible working conditions, laboratory protocols, and readout assays underlined this hypothesis. We were able to provide examples for functional heterogeneity of mammalian innate immune cell populations in their in vitro response against the pathogenic mold A. fumigatus. These specific differences should be carefully considered in future comparative discovery and validation studies. Furthermore, they highlight potential limitations in the direct transferability of murine host-pathogen interaction studies. Additional studies are highly warranted comparatively employing murine and human cell populations to further study mold immunopathology and to evaluate new diagnostic and therapeutic strategies. Moreover, work on humanized mouse models or the development of synthetic human models might help to avoid difficulties in translating and harmonizing data across different mammalian species.

\section{ETHICS STATEMENT}

Use of whole blood specimens from healthy volunteers was approved by the University Hospital of Wuerzburg Ethical Committee (\#302/15). Written informed consent was obtained and data analysis was performed anonymously. Mouse husbandry and experimental procedures were conducted in accordance with institutional guidelines and with the approval of the Committee on Animal Research of the regional government (Regierung von Unterfranken, Wuerzburg, Germany).

\section{AUTHOR CONTRIBUTIONS}

A-MH and JaL designed the study, performed experiments, performed data analyzes, and wrote the manuscript. AS performed

\section{REFERENCES}

1. Segal BH. Aspergillosis. N Engl J Med (2009) 360:1870-84. doi:10.1056/ NEJMra0808853

2. Grimm MJ, D’Auria AC, Segal BH. Assessing anti-fungal activity of isolated alveolar macrophages by confocal microscopy. J Vis Exp (2014) (89):e51678. doi: $10.3791 / 51678$

3. Braedel S, Radsak M, Einsele H, Latge JP, Michan A, Loeffler J, et al. Aspergillus fumigatus antigens activate innate immune cells via toll-like receptors 2 and 4. Br J Haematol (2004) 125:392-9. doi:10.1111/j.1365-2141. 2004.04922.x

4. MezgerM,KneitzS, WozniokI, KurzaiO, EinseleH,LoefflerJ. Proinflammatory response of immature human dendritic cells is mediated by Dectin-1 after exposure to Aspergillus fumigatus germ tubes. J Infect Dis (2008) 197:924-31. doi:10.1086/528694

5. Mestas J, Hughes CC. Of mice and not men: differences between mouse and human immunology. J Immunol (2004) 172:2731-8. doi:10.4049/ jimmunol.172.5.2731

6. Reynolds G, Haniffa M. Human and mouse mononuclear phagocyte networks: a tale of two species? Front Immunol (2015) 6:330. doi:10.3389/ fimmu.2015.00330 experiments. SW and ML analyzed the data and contributed to the manuscript. $\mathrm{CM}, \mathrm{ME}$, and $\mathrm{KC}$ provided discussions, technical assistance and contributed to the manuscript. HE and JuL developed concepts, supervised the study, and wrote the manuscript.

\section{FUNDING}

Thisstudywassupported bytheDeutscheForschungsgemeinschaft (DFG) within the Collaborative Research Center CRC124 FungiNet "Pathogenic fungi and their human host: Networks of interaction" (project A2 to $\mathrm{HE}$ and JuL, A4 to JaL and funding for travel to $\mathrm{CM}$ ) and by the Interdisciplinary Centre for Clinical Research Wuerzburg (grant Z-3/56 to SW). The authors thank all blood donors for their voluntary donation.

\section{SUPPLEMENTARY MATERIAL}

The Supplementary Material for this article can be found online at http://www.frontiersin.org/article/10.3389/fimmu.2017.01716/ full\#supplementary-material.

FIGURE S1 | Comparison of cytokine response patterns to Aspergillus fumigatus germ tubes. Cytokine secretion into the culture medium was analyzed by multiplex bead-based assay upon stimulation of murine or human dendritic cells (DCs), neutrophils [polymorphonuclear cells (PMNs)], or macrophages with A. fumigatus germ tubes. Fold changes compared to unstimulated samples are indicated by gray scale. OOR (out of range) indicates that $>50 \%$ of samples did not meet the limits of detection and thus fold changes could not be calculated reliably.

FIGURE S2 | Impact of human and murine polymorphonuclear cells (PMNs) on fungal XTT metabolism. PMNs were isolated from whole blood of four healthy human donors and two mice as described in the Section "Materials and Methods." After 2 and $4 \mathrm{~h}$ of coculture at $37^{\circ} \mathrm{C}$, hypotonic lysis of PMNs was performed. Supernatants were carefully removed and $200 \mu \mathrm{HBSS}$ supplemented with $400 \mu \mathrm{g} / \mathrm{ml}$ of 2,3-bis-(2-methoxy-4-nitro-5-sulphenyl)-(2 H)tetrazolium-5-carboxanilide (XTT) and $50 \mu \mathrm{g} / \mathrm{ml}$ of coenzyme were added. After $90 \mathrm{~min}$ incubation at $37^{\circ} \mathrm{C}$ and centrifugation at $300 \mathrm{~g}$ for $5 \mathrm{~min}, 100 \mu \mathrm{l}$ supernatant of each well were transferred to a 96 -well plate and $\mathrm{OD}_{450}$ was measured in a microplate reader. Fold changes of fungal XTT metabolism due to the presence of PMNs was calculated according to the following formula: Fold change $=\left(\mathrm{OD}_{450}^{\text {PMN F Fungus }}-O D_{450}^{\text {PMN }}\right) /\left(O D_{450}^{\text {Fingus }}-O D_{450}^{\text {Bank }}\right)$. Individual results for each human and murine PMN sample and mean values (black horizontal bars) are shown in the figure.

7. Relle M, Thomaidis T, Galle PR, Schwarting A. Comparative aspects of murine proteinase 3. Rheumatol Int (2011) 31:1105-11. doi:10.1007/s00296010-1672-2

8. Park SJ, Burdick MD, Mehrad B. Neutrophils mediate maturation and efflux of lung dendritic cells in response to Aspergillus fumigatus germ tubes. Infect Immun (2012) 80:1759-65. doi:10.1128/IAI.00097-12

9. Lother J, Breitschopf T, Krappmann S, Morton CO, Bouzani M, Kurzai O, et al. Human dendritic cell subsets display distinct interactions with the pathogenic mould Aspergillus fumigatus. Int J Med Microbiol (2014) 304:1160-8. doi:10.1016/j.ijmm.2014.08.009

10. Moretti S, Bozza S, Massi-Benedetti C, Prezioso L, Rossetti E, Romani L, et al. An immunomodulatory activity of micafungin in preclinical aspergillosis. J Antimicrob Chemother (2014) 69:1065-74. doi:10.1093/jac/dkt457

11. Prufer S, Weber M, Stein P, Bosmann M, Stassen M, Kreft A, et al. Oxidative burst and neutrophil elastase contribute to clearance of Aspergillus fumigatus pneumonia in mice. Immunobiology (2014) 219:87-96. doi:10.1016/j. imbio.2013.08.010

12. Liu C, Wang M, Sun W, Cai F, Geng S, Su X, et al. PU.1 serves a critical role in the innate defense against Aspergillus fumigatus via dendritic cell-associated C-type lectin receptor-1 and toll-like receptors-2 and 4 in THP-1-derived macrophages. Mol Med Rep (2017) 15:4084-92. doi:10.3892/mmr.2017.6504 
13. Wiemann P, Perevitsky A, Lim FY, Shadkchan Y, Knox BP, Landero Figueora JA, et al. Aspergillus fumigatus copper export machinery and reactive oxygen intermediate defense counter host copper-mediated oxidative antimicrobial offense. Cell Rep (2017) 19:2174-6. doi:10.1016/j.celrep.2017.05.075

14. Lutz MB, Kukutsch N, Ogilvie AL, Rossner S, Koch F, Romani N, et al. An advanced culture method for generating large quantities of highly pure dendritic cells from mouse bone marrow. JImmunol Methods (1999) 223:77-92. doi:10.1016/S0022-1759(98)00204-X

15. Sales-Campos H, Tonani L, Cardoso CR, Kress MR. The immune interplay between the host and the pathogen in Aspergillus fumigatus lung infection. Biomed Res Int (2013) 2013:693023. doi:10.1155/2013/693023

16. Doeing DC, Borowicz JL, Crockett ET. Gender dimorphism in differential peripheral blood leukocyte counts in mice using cardiac, tail, foot, and saphenous vein puncture methods. BMC Clin Pathol (2003) 3:3. doi:10.1186/ 1472-6890-3-3

17. Haley PJ. Species differences in the structure and function of the immune system. Toxicology (2003) 188:49-71. doi:10.1016/S0300-483X (03)00043-X

18. Zschaler J, Schlorke D, Arnhold J. Differences in innate immune response between man and mouse. Crit Rev Immunol (2014) 34:433-54. doi:10.1615/ CritRevImmunol.2014011600

19. Arnhold J, Flemmig J. Human myeloperoxidase in innate and acquired immunity. Arch Biochem Biophys (2010) 500:92-106. doi:10.1016/j.abb. 2010.04.008

20. Brakhage AA, Bruns S, Thywissen A, Zipfel PF, Behnsen J. Interaction of phagocytes with filamentous fungi. Curr Opin Microbiol (2010) 13:409-15. doi:10.1016/j.mib.2010.04.009

21. Shlezinger N, Irmer H, Dhingra S, Beattie SR, Cramer RA, Braus GH, et al. Sterilizing immunity in the lung relies on targeting fungal apoptosis-like programmed cell death. Science (2017) 357:1037-41. doi:10.1126/science. aan0365

22. Zlotnik A, Yoshie O, Nomiyama H. The chemokine and chemokine receptor superfamilies and their molecular evolution. Genome Biol (2006) 7:243. doi: 10.1186/gb-2006-7-12-243

23. Rossi DL, Hurst SD, Xu Y, Wang W, Menon S, Coffman RL, et al. Lungkine, a novel CXC chemokine, specifically expressed by lung bronchoepithelial cells. J Immunol (1999) 162:5490-7.

24. Helft J, Bottcher J, Chakravarty P, Zelenay S, Huotari J, Schraml BU, et al. GM-CSF mouse bone marrow cultures comprise a heterogeneous population of $\mathrm{CD} 11 \mathrm{c}(+) \mathrm{MHCII}(+)$ macrophages and dendritic cells. Immunity (2015) 42:1197-211. doi:10.1016/j.immuni.2015.05.018

25. Hettinger J, Richards DM, Hansson J, Barra MM, Joschko AC, Krijgsveld J, et al. Origin of monocytes and macrophages in a committed progenitor. Nat Immunol (2013) 14:821-30. doi:10.1038/ni.2638

26. Romani N, Gruner S, Brang D, Kampgen E, Lenz A, Trockenbacher B, et al. Proliferating dendritic cell progenitors in human blood. JExp Med (1994) 180:83-93. doi:10.1084/jem.180.1.83

27. Randolph GJ, Beaulieu S, LebecqueS, Steinman RM, Muller WA. Differentiation of monocytes into dendritic cells in a model of transendothelial trafficking. Science (1998) 282:480-3. doi:10.1126/science.282.5388.480

28. Cheong C, Matos I, Choi JH, Dandamudi DB, Shrestha E, Longhi MP, et al. Microbial stimulation fully differentiates monocytes to DC-SIGN/ CD209(+) dendritic cells for immune T cell areas. Cell (2010) 143:416-29. doi:10.1016/j.cell.2010.09.039

29. Xu Y, Zhan Y, Lew AM, Naik SH, Kershaw MH. Differential development of murine dendritic cells by GM-CSF versus Flt3 ligand has implications for inflammation and trafficking. J Immunol (2007) 179:7577-84. doi:10.4049/ jimmunol.179.11.7577

30. Segura E, Touzot M, Bohineust A, Cappuccio A, Chiocchia G, Hosmalin A, et al. Human inflammatory dendritic cells induce Th17 cell differentiation. Immunity (2013) 38:336-48. doi:10.1016/j.immuni.2012.10.018

31. Steinman RM, Hemmi H. Dendritic cells: translating innate to adaptive immunity. Curr Top Microbiol Immunol (2006) 311:17-58.

32. Maree AF, Komba M, Finegood DT, Edelstein-Keshet L. A quantitative comparison of rates of phagocytosis and digestion of apoptotic cells by macrophages from normal $(\mathrm{BALB} / \mathrm{c})$ and diabetes-prone (NOD) mice. JAppl Physiol (1985) (2008) 104:157-69. doi:10.1152/japplphysiol.00514. 2007

33. Cenci E, Mencacci A, Casagrande A, Mosci P, Bistoni F, Romani L. Impaired antifungal effector activity but not inflammatory cell recruitment in interleukin-6-deficient mice with invasive pulmonary aspergillosis. J Infect Dis (2001) 184:610-7. doi:10.1086/322793

34. Brieland JK, Jackson C, Menzel F, Loebenberg D, Cacciapuoti A, Halpern J, et al. Cytokine networking in lungs of immunocompetent mice in response to inhaled Aspergillus fumigatus. Infect Immun (2001) 69:1554-60. doi:10.1128/ IAI.69.3.1554-1560.2001

35. Windsor WT, Syto R, Tsarbopoulos A, Zhang R, Durkin J, Baldwin S, et al. Disulfide bond assignments and secondary structure analysis of human and murine interleukin 10. Biochemistry (1993) 32:8807-15. doi:10.1021/ bi00085a011

36. Willment JA, Marshall AS, Reid DM, Williams DL, Wong SY, Gordon S, et al. The human beta-glucan receptor is widely expressed and functionally equivalent to murine Dectin-1 on primary cells. Eur J Immunol (2005) 35:1539-47. doi:10.1002/eji.200425725

37. Brown GD, Herre J, Williams DL, Willment JA, Marshall AS, Gordon S. Dectin-1 mediates the biological effects of beta-glucans. J Exp Med (2003) 197:1119-24. doi:10.1084/jem.20021890

38. Reid DM, Gow NA, Brown GD. Pattern recognition: recent insights from Dectin-1. Curr Opin Immunol (2009) 21:30-7. doi:10.1016/j.coi.2009.01.003

39. Hefter M, Lother J, Weiß E, Schmitt AL, Fliesser M, Einsele H, et al. Human primary myeloid dendritic cells interact with the opportunistic fungal pathogen Aspergillus fumigatus via the C-type lectin receptor Dectin-1. Med Mycol (2016) 55:573-8. doi:10.1093/mmy/myw105

40. Werner JL, Metz AE, Horn D, Schoeb TR, Hewitt MM, Schwiebert LM, et al. Requisite role for the Dectin-1 beta-glucan receptor in pulmonary defense against Aspergillus fumigatus. J Immunol (2009) 182:4938-46. doi:10.4049/ jimmunol.0804250

41. Raberg L, Sim D, Read AF. Disentangling genetic variation for resistance and tolerance to infectious diseases in animals. Science (2007) 318:812-4. doi:10.1126/science.1148526

Conflict of Interest Statement: The authors declare that the research was conducted in the absence of any commercial or financial relationships that could be construed as a potential conflict of interest.

Copyright $\odot 2017$ Hellmann, Lother, Wurster, Lutz, Schmitt, Morton, Eyrich, Czakai, Einsele and Loeffler. This is an open-access article distributed under the terms of the Creative Commons Attribution License (CC BY). The use, distribution or reproduction in other forums is permitted, provided the original author(s) or licensor are credited and that the original publication in this journal is cited, in accordance with accepted academic practice. No use, distribution or reproduction is permitted which does not comply with these terms. 